\title{
Oralité et préservation de la mémoire par l'écriture
}

\section{Résumé:}

Dans cet article, nous nous intéressons à la manière dont Latifa Ben Mansour adapte l'oralité aux codes de l'écriture dans La Prière de la peur. Les modes de l'oralité et de l'écriture étant différents, l'introduction de l'oralité dans le texte romanesque s'accompagne de procédés et de pratiques spécifiques. En mettant l'accent sur les mécanismes d'intégration de l'oralité dans le texte littéraire, notre analyse revientsur les modalités de restitution de l'oralité dans et par le texte mansourien et le rôle joué par la construction textuelle dans la préservation de la mémoire et sa transmission.

Mots clés : oralité, écriture, mémoire, transmission, personnages.

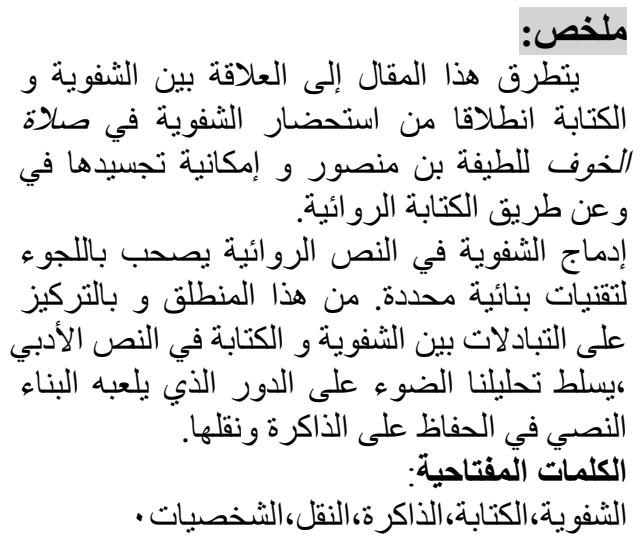

Inès BOUKEZOULA

Département des lettres et langue française Université des frères Mentouri Constantine 1

\begin{abstract}
Introduction:
Plusieurs chercheurs à travers le monde ont tiré la sonnette d'alarme pour attirer l'attention sur la déperdition dont fait l'objet au quotidien le patrimoine culturel essentiellement oral, et sensibiliser toutes las catégories à la nécessité de sa préservation.
\end{abstract}


Sensible à cette problématique, Latifa Ben Mansour écoute et restitue dans ses écrits la tradition orale parles voix maternelles. L'auteure explique : Je voulais par l'écriture poursuivre cette tâche féminine de la tradition orale. Je souhaitais parler de notre culture, de tout ce passé qui est un des plus riches de l'histoire de l'humanité et qu'on est en train de faire disparaître. ${ }^{(1)}$

Par la voix du personnage principal de La Prière de la peur ${ }^{(2)}$, Ben Mansour déclare : Les conteurs ne sont plus et les artisans sont morts ou passés à «l'industrie industrialisante ». (p. 321).

Les premières civilisations assuraient la continuité de la mémoire collective par le biais de la transmission orale. Les garants de cette continuité avaient à charge de perpétuer la tradition qui servait de modèle de conduite aux générations futures. La mémoire restait le pilier de cette transmission. Mais face aux mutations sociales et technologiques, l'oralité se trouve de plus en plus menacée d'où l'urgence de la fixer sur des supports matériels durables. Basée sur la transmission d'une part, le partage et la rediffusion de l'autre :La tradition orale appelle l'écriture à son secours pour se perpétuer ${ }^{(3)}$,comme l'affirme Michel Butor.

En intégrant l'oralité dans le roman, Ben Mansour combine deux modes communicationnels distincts. Le passage de l'oral à l'écrit s'accompagne de variations discursives et énonciatives telles que l'absence de la dimension matérielle de la voix humaine, de l'intonation du locuteur, de sa présence physique. Quels mécanismes permettent à l'auteurede reconstituer une réalité immédiate et insaisissable sur un support par essence médiat ? La construction textuelle est-elle à même de restituer le caractère vivant de l'oralité et de contribuer ainsi à sa sauvegarde et à sa transmission ?

Nous essayerons de répondre à ces interrogations en prenant appui sur La Prière de la peur, roman de Latifa Ben mansour.

Paru en 1997, le récit raconte l'histoire d'un personnage dédoublé prénommé Hanan. Victime d'un attentat à l'aéroport d'Alger et sachant que ses jours sont comptés, Hanan se réfugie à Aïn $\mathrm{Al}$ Hout, son village natal, et se lance dans une course contre la montre pour transcrire toutes les histoires racontées par l'aïeule, Lalla Kenza.La jeune femme demande à ses proches de lui apporter les manuscrits de la famille(p. 27) pour qu'elle achève son travail de mémoire : Transportez-moi à Aïn el Hout et Lalla Kenza restera auprès de moi. J'ai besoin d'elle. Elle me transmettra ce que je ne trouverai pas dans les livres et les manuscrits.(p. 27).

Gardienne de la mémoire collective, Lalla Kenza a dans le récit le rôle de personnage conteur. Élément clé du roman, Lalla Kenza dont le prénom signifie «trésor : kenz », accompagne les deux protagonistes du récit, elle est : Le pilier indestructible (...) le roc auquel on se cramponne dans la tourmente et le désespoir, le socle et l'assise de la famille et du pays, (p. 23). L'aïeule est le 


\section{Oralité et préservation de la mémoire par l'écriture}

puits de savoir qui veillera sur Hanan, sa petite-fille, durant ses derniers mois de vie, (...) elle me transmettra ce que je ne trouverai pas dans les livres et les manuscrits, explique la jeune femme, (p. 27), et l'aidera à achever son manuscrit, dans leur cellule, (p. 25), près du mausolée de Sidi Mohammed Ben Ali à Ain el Hout, la terre de ses ancêtres.

La presque centenaire grand-mère prend souvent la parole dans le roman se chargeant de transmettre à la jeune femme l'histoire de la famille, ponctuée du chant des descendants des sept coupoles, (p. 28). Elle lui transmet son savoir, Hanan écrivait sans s'arrêter, (p. 28), et (...) se laissait prendre à sa manière de transmettre. (p. 31).

Lalla raconte à Hanan les légendes des ancêtres, elle est le trésor où se trouve préservée la mémoire collective. Adoptant une démarche didactique, l'aïeule explique à sa petite-fille le pourquoi de certains rites, traditions ou croyances,

En elle logeaient plusieurs livres. Ceux qu'elle avait appris par cœur, pour les soustraire aux autodafés, et ceux qu'elle avait inventés (...). Elle avait retenu des milliers de pages comme les magiciens, elle attendait la nuit, une oreille respectueuse et bienveillante pour sortir de sa bouche, comme un illusionniste ferait sortir des lames de rasoir, les mots tréfilés en une longue chaîne sonore. Des sons mélodieux devenant un magnifique et féérique fil de soie. (p.30).

Les récits de l'aïeule transportent Hanan loin de sa tragédie et lui font oublier sa mort imminente. Lorsque Lalla Kenza se mettait à raconter, une chaleur invisible parcourait les auditeurs. Cette chaleur était source de vie, (p. 31), et donnait à Hanan l'inspiration et le courage nécessaires pour rédiger son manuscrit, et conserver ainsi pour toujours ce que lui transmettait Lalla :

Aucun livre n'aurait la saveur ni les couleurs des personnages racontés par Lalla Kenza. Dire qu'elle était à elle seule une bibliothèque, serait une image inodore, incolore. Il y avait la richesse des termes qu'elle utilisait, le ton qu'elle prenait, les chants qu'elle pouvait entonner sur n'importe quel mode, les rires qui fusaient ou les larmes et sanglots qui pouvaient inonder son visage comme si elle était elle-même le héros ou l'héroïne. (p. 31)

Lalla ne se contentait pas seulement de raconter à Hanan des contes, des légendes ou l'histoire familiale, elle se chargeait également de lui expliquer leur signification, préconisant (...) à celui qui l'entendait de savoir retrouver le chemin de la lumière, (p. 30). C'est l'aïeule qui explique le titre du roman à sa petite fille :

La prière de la peur ne se récite pas, ma fille. C'est un rituel instauré par le prophète Mohammad pour conjurer la peur. Un 
prophète aussi peut éprouver de la frayeur, être traversé de doutes. Mohammad sur Lui la Paix et le Salut, n'avait pas honte d'avouer son anxiété et son épouvante face à la mort, ma fille, il n'avait cessé de répéter : "Je ne suis qu'un homme ». C'est cela le sens de cette prière. (p. 56).

De cette explication se dégage une volonté de la part de l'aïeule de préparer sa petite-fille à affronter la peur de sa propre mort, à préparer son départ imminent.

Les échanges entre Hanan et Lalla Kenza sont construits à la manière d'un cours où la jeune femme, avide de savoir, pose continuellement des questions à l'aïeule qui les lui explique : Pourquoi [raconter des histoires] la nuit ? demandait Hanan avec curiosité (p. 30) ; Peux-tu m'expliquer, Lalla, pourquoi les poissons et le lion sont le blason des descendants des sept coupoles ? (p. 42). La vénérable dame enchaîna par une autre histoire(p. 46) ;Connais-tu l'histoire des trois mères et des trois soupirs ?, (p. 51). Le soir de sa mort, Hanan (...) demanda à Lalla Kenza de lui raconter l'histoire du SULTAN BIEN-AIMÉ (...) Elle attendait avec l'impatience du dernier souffle que l'aïeule se mit à parler. (p. 81).

Les contes et légendes relatés de mémoire par Lalla ont une valeur essentiellement collective, de par leur contenu, puisqu'ils se rapportent majoritairement à l'histoire des ancêtres, mais aussi parce qu'ils ont été transcrits par la petite-fille dans un manuscrit censé les préserver définitivement de l'oubli et de la déperdition : Hanan buvait lentement son café noir, puis elle dirigeait sa chaise roulante vers la table de travail, et transcrivait l'histoire racontée la veille par l'aïeule (p. 41). L'écriture se fait dans la douleur et l'empressement, Hanan sentant de jour en jour que sa fin est de plus en plus proche.

La jeune femme passedonc ses derniers mois à écrire sans s'arrêter. $\mathrm{Ce}$ personnage scripteur s'efface au moment où est introduit le personnage lecteur, une cousine appartenant à la nouvelle génération. En effet, àla mort de Hanan, le manuscrit est confié par Lalla Kenza à sa petite-fille qui porte le même prénom que la défunte pour qu'elle le lise devant toute la famille lors de sa veillée funèbre.La lectrice vivait aussi en exil à Fès, c'est la première fois qu'elle rentre en Algérie après plusieurs années d'absence répondant ainsi à l'appel de la mémoire.

La lecture publique du manuscrit donne l'occasion à l'auditoire de prendre la parole et toute l'assistance se transforme en conteurs et conteuses qui relatent des histoires à tour de rôle se détachant ainsi de l'évènement qui les rassemble, la veillée funèbre, et s'unissant non pas pour célébrer la mort, mais plutôt autour du manuscrit et de la mémoire collective qui y est contenue.

Le manuscrit joue le rôle du conte qui introduit son auditeur ou son lecteur dans un monde imaginaire qui lui fait oublier, le temps de la narration, le monde réel en le transportant dans un ailleurs lointain, tout en stimulant son imagination. Rassemblés autour du manuscrit de la défunte lu par l'exilée, les 
auditeurs, interpellés par le patrimoine commun contenu dans le texte de Hanan, prennent à tour de rôle la parole, cette lecture publique entraîne (...) chacun à se lire, c'est-à-dire à inventer soi-même sa vie. ${ }^{(4)}$

Lorsqu'un conte esténoncé dans le monde réel, les formules d'entrée et de sortie sont le moyen de revenir vers ce monde. Mais quand il s'agit d'un conte introduit dans la trame narrative d'un roman, ces formules sont l'outil qui permet d'une part, la simulation d'une situation réelle au cœur du récit, celle d'un conte raconté devant un auditoire (les personnages) et offre d'autre part la possibilité de créer une diversité narrative dans le roman. On ne revient pas à la fin du conte vers le monde réel, mais vers le monde réel des personnages du roman. L'œuvre est une superposition de mondes, celui du réel qui inspire l'auteure, celui de la fiction créé par la production romanesque elle-même et enfin celui de l'imaginaire représenté par le conte.

Échappant à toute temporalité et à toute localisation, le manuscrit constitue une parenthèse qui s'ouvre dans l'espace textuel. Marqué typographiquement par l'utilisation des guillemets et de l'italique, le récit du manuscrit se déroule comme un conte en reprenant dès son énonciation ses formules d'ouverture et de fin. Le recours à ces formules propres aux conte crée une distance entre le monde auquel ils appartiennent et l'espace textuel romanesque. Le conte se superpose de ce fait au roman tout en se situant en dehors de son monde, il a ses propres personnages différents de ceux du récit dans lequel il est inséré, des évènements qui lui sont propres. C'est une «forme close », comme le présente Georges Jean ${ }^{(5)}$, greffée à une autre ouverte. C'est un monde second au sein de l'œuvre romanesque qui s'y présente comme un second plan narratif, et participe ainsi à son éclatement tout en contribuant à la diversité narrative et romanesque.

Les contes repris dans le manuscrit de Hanan constituent aussi des pauses dans le récit-cadre. C'est une échappatoire qui permet aux personnages de fuir momentanément la violence de la réalité. Espace d'évasion, il est pour les personnages et le lecteur comme une page publicitaire qui interrompt momentanément le récit d'évènements.

L'espace est également fractionné entre passé et présent, entre un icimaintenant dans lequel les protagonistes ne se reconnaissent plus, et un ailleurs inscrit dans le passé, marqué par la nostalgie, voir la désillusion. Le cercle constitué autour du personnage lecteur par les membres de la famille lors de la lecture du manuscrit à la veillée funèbre reprend la tradition de la halqa et marque une distance entre l'espace-temps constitué par ce cercle et l'espacetemps du récit-cadre.

Comme s'ils étaient dans un conte, les personnages de ce roman se dissocient du présent, ils lui préfèrent un passé habité par des lieux qui n'existent plus que dans leur mémoire et quimarquent leur enracinement, leur attachement aux sources et aux origines, reprenant une caractéristique des contes qui : Se 
déroulent dans le passé, mais ce passé ne ressemble pas à celui de l'Histoire : il est sans date et sans réalité. Les premiers mots du conte renvoient au temps des origines, au monde perdu auquel nous n'avons plus accès. ${ }^{(6)}$

La ville de Tlemcen est évoquée par ses anciennes appellations, comme si les personnages voulaient fuir le présent et continuer à vivre dans un temps qui n'existe plus.Lalla Kenza, la première diseuse d'histoires du récit, vit pleinement dans ce passé qu'elle restitue comme une conteuse, inscrivant le lecteur (...) dans une chronologie nostalgique, écho d'un temps durable et révolu où sans doute était possible ce qui n'a plus cours aujourd'hui. ${ }^{(7)}$

Ainsi conçue, l'écriture mansourienne fractionne le discours. Le romanmansouriense construit autour d'un récit-cadre dans lequel sont enchâssés d'autres récits pris en charge par différents personnages, ce procédé participerait à l'éclatement narratif ainsi qu'à la mise en place de la polyphonie narrative. Les personnages racontent et se racontent, désarticulant ainsi la linéarité textuelle.

Latifa Ben Mansour reprend ainsi la technique d'enchâssement et des récits emboités marchant sur les traces des Mille et une nuits. Stimulée par la lecture du manuscrit, la parole est partagée entre plusieurs narrateurs qui racontent leurs expériences et leur savoir culturel afin de les partager et de les transmettre. L'espace romanesque permet de ce fait la conservation de l'authenticité culturelle, il offre la possibilité d'une réutilisation d'éléments appartenant à la culture et la littérature orales que le texte romanesque actualise. Le récit prend alors la forme de contes superposés et entremêlés où la parole se ballade de personnage en personnage.

Deux plans narratifs se combinent au cœur de la fiction : une sorte de fiction au sein de la fiction. L'introduction d'un manuscrit dans le récit premier peut être considérée comme un cadre narratif second, le récit de par sa narration, devient ainsi double. L'auteure ne se contente pas d'introduire des contes mais procède à un travail stylistique qui met en jeux diverses composantes du conte.

Par cette répartition de la parole et la création de personnages conteur, scripteur et lecteur, la préservation et la transmission du patrimoine collectif est assurée, et le respect de la chaîne de transmission, allant de la personne la plus âgée à la plus jeune, est pris en compte. La triade transmission-préservationretransmission est observéedans ce roman, malgré la mort qui guette les trois personnages assurant cette mission.

Le conte rassemble, tout l'auditoire est suspendu aux lèvres du conteur : Le moment du conte est un moment de respiration commune. ${ }^{(8)} \mathrm{C}$ 'est pour cette raison que le dernier désir de Hanan fut que son manuscrit, qui débute par un conte et est écrit à la manière d'un conte, soit lu la nuit, devant toute la famille. La lecture du manuscrit prend ainsi la forme d'une veillée familiale où chacun dans l'assistance prend du plaisir à relater des contes et tous les personnages se muent en conteurs. 
La parole est partagée entre ces différentes voix, appartenant à différentes générations, inscrivant le récit dans un contexte pluriel, à la fois polyphonique et dialogique. Le dialogisme implique une pluralité au niveau idéologique, l'auteur n'est pas le seul idéologue dans le roman, il rend compte de cette pluralité en multipliant les voix dans le récit. Tout discours est par essence dialogique, il est constitué par rapport à un déjà-dit : Seul l'Adam mythique abordant avec sa première parole un monde pas encore mis en question, vierge, seul Adam-le solitaire pouvait éviter totalement cette orientation dialogique sur l'objet avec la parole d'autrui. ${ }^{(9)}$

Ainsi, narrateurs et personnages expriment aussi leurs idées, qu'elles soient similaires,proches, ou complètement différentes et opposées à celles de l'auteur.Pour assurer l'autonomie des consciences, ces points de vue doivent être représentés par des instances discursives différentes (narratives dans le cadre du texte littéraire) et non pas par une seule instance représentative et porteuse de la voix de l'auteur. La polyphonie impose un éloignement du cadre monologique, lequel implique l'unicité.

L'hybridation dans le roman polyphonique apparait dans la distinction narrateur/ personnage, le narrateur devient perméable au style des personnages, l'instance narratoriale projette les personnages - à travers l'échange- dans l'émancipation dialogique.Le personnage ne porte pas seulement un mot (discours) sur lui-même ou sur les autres, il observe et interprète tout ce qui l'entoure, il a son propre point de vue sur le monde.Pour Bakhtine, la polyphonie littéraire dépasse la pluralité de voix, c'est aussi une pluralité de consciences autonomes et d'univers idéologiques ; il note que le personnage (...) n'est pas seulement une conscience, il est surtout idéologue. ${ }^{(10)}$

Bakhtine considère, dans ce sens, que le point de vue narratif et la vérité qu'a le personnage sur le monde sont étroitement liés à la vérité personnelle de ce dernier. Il insiste sur la personnification des idées. L'idée est d'autant plus le point nodal entre dialogisme et monologisme. Le théoricien russe affirme qu'une interaction verbale entre consciences autonomes ne peut avoir lieu dans une perception monologique où les idées appartiennent à l'auteur qui les exprime sans les confronter à d'autres points de vue contraires.

L'auteur écrit, imagine, organise ou invente les évènements, il introduit le narrateur qui les rapporte. C'est la voix de la fiction qui raconte, décrit, commente, informe ou interprète :

Ce qui importe à l'auteur, ce n'est pas une façon typique et individuelle de penser, de sentir, de parler, mais avant tout une manière de voir et de représenter : c'est le but essentiel du remplacement de l'auteur par le narrateur. ${ }^{(11)}$ 
La prière de la peur est également construit sur ce principe de pluralité vocale, où la parole est donnée, tantôt à tour de rôle, tantôt simultanément, à plusieurs personnages féminins. Il faut signaler d'emblée que 16 des 23 chapitres qui composent le récit sont majoritairement construits de récits de paroles.

Le récit s'ouvre avec Abla, la mère de la protagoniste qui exprime d'entrée de jeu son malaise à l'encontre de certains comportements étrangers à la société algérienne durant les années 90 . Cette femme perd, hélas, la raison quand elle apprend que sa fille est victime de l'attentat de l'aeroport d'Alger, elle devient absente au monde et se mure dans le silence.

Le récit est ensuite rythmé aux sons des femmes qui le tissent. La parole est alors confiée à Lalla Kenza tout au long des chapitres 3-4-6 et 7, pour raconter l'histoire de la famille et celle des ancêtres. Ce récit est néanmoins entrecoupé de questions et de commentaires de la protagoniste, Hanan, qui transcrit en même temps les paroles de l'aïeule dans son manuscrit.

Le septième chapitre a une importance particulière dans le récit, il comporte la plus longue histoire racontée dans le roman, celle de Ruh Al Aghrib. Cette légende locale est racontée par Lalla Kenza, elle est d'une diversité particulière ; l'auteure y fusionne langue arabe et langue française, versets coraniques et poèmes, mais également des explications de mots en arabe classique. C'est à la fin de ce chapitre que Ben Mansour a choisi de passer de la parole : le conte raconté par l'aïeule, à l'événement : la mort de la protagoniste. La disparition de cette première narratrice dans le récit permet à l'auteure d'introduire la voix de la jeune Hanan.

La narration du chapitre 5 est assumée par ailleurs par un personnage haut en couleurs, Al Hamra, Une conteuse et une imitatrice de premier ordre ! (p.355), qui reprend la parole un peu plus loin vers la fin du récit dans le chapitre 22, lors de la veillée funèbre de la protagoniste. Al Hamra (...) était à elle seule toutes les Shéhérazade ! (p.356), elle est connue pour son interprétation majestueuse de contes et de légendes, mais également pour son franc-parler.

Les chapitres 9 et 10 constituent quant à eux une sorte de pauses dans le récit. Un narrateur extradiégétique, mais omniscient, y relate l'arrivée de la jeune Hanan à Ain el Hout pour assister aux funérailles de sa cousine, c'est à ce moment-là qu'elle prend connaissance de la mission que lui a confié la défunte : lire son manuscrit devant toute la famille.

Les chapitres 10-11-12-17-18 et 19 sont lus à partir du manuscrit par l'autre Hanan, la jeune cousine, narratrice extradiégétique dans un premier temps. Cette lecture n'est nullement linéaire, elle est entrecoupée des commentaires de la lectrice, des recommandations de l'aïeule Lalla Kenza, mais aussi d'interventions des membres de la famille présents lors de la veillée funèbre, qui «faisaient cercle » (p. 191) autour de la lectrice transformée en conteuse. 
La lecture du manuscrit pousse inconsciemment la jeune Hanan à raconter sa propre histoire tout au long des chapitres 13-14-15 et 16, et à confondre son parcours, sa voix et même sa personne avec sa cousine décédée. On assiste ici à une superposition des voix narratives, Hanan passe de narratrice extradiégétique à narratrice intradiégétique et de narratrice hétérodiégétique à narratrice homodiégétique.

Pour Berthelot, le narrateur homodiégétique représenté dans le récit par Hanan, le personnage transcripteur,(...) s'exprime à deux niveaux : écrit en tant que narrateur, et oral en tant que personnage. ${ }^{(12)}$

Le narrateur autodiégétique, Hanan la lectrice du manuscrit, (...) est constamment amené à retransmettre ses paroles ${ }^{(13)}$, produisant un effet polyphonique à travers ce dédoublement des voix, et dialogique, puisque chacune d'entre elles est constituée indépendamment de celle qui l'a engendrée.

Les chapitres 20 et 21 constituent pour leur part une pause dans le récit, Hanan décide d'interrompre la lecture du manuscrit pour faire visiter Tlemcen à son mari en plein nuit.

Les événements se précipitent ensuite tout au long des deux derniers chapitres, Hanan reprend avec grande peine la lecture du manuscrit. Elle ne s'arrête que pour laisser place à un ultime récit fabuleux d'Al Hamra, à la complainte du luth de Moulay, ou aux exclamations des femmes qui l'entourent.

Le fait de confier la narration à autant de femmes participe à la pluralité narrative et vocale du récit, à la multiplication des perspectives et des points de vue narratifs, mais surtout à l'instauration d'une chaîne de parole qui assure la transmission de la parole ancestrale, légendaire et fabuleuse, dans un contexte historique marqué par la violence.

En adoptant ce mécanisme de pluralité vocale, Ben Mansour insiste sur l'importance de la prise de parole et de sa transmission. La parole de Lalla Kenza est transcrite dans un manuscrit lu par la jeune Hanan en présence de toute la famille, qui le commente en même temps, et dont le contenu invoque chez chaque personnage présentlors de la veillée funèbre un souvenir qu'il racontera à son tour. La voix de Hanan éteinte par la mort est plus que jamais audible, elle provoque à contrario la profusion d'autres voix dans le récit.

La transmission orale est renforcée par le choix de la lectrice du manuscrit, une jeune femme portant le même prénom que celle qui l'a rédigé, pour marquer la continuité entre les générations. Hanan devait lire le manuscrit devant toute la famille, dans la cours de la maison familiale, entourée des membres de la famille qui forment un cercle autour d'elle. Ces conditions rappellent celles de l'énonciation des contes d'antan sur les places publiques.

L'acte d'écouter, d'écrire et de narrer sont mis en avant. Le premier personnage, Hanan, passe d'auditeur à scripteur d'un manuscrit qui convoque une lectrice préalablement désignée, pour transformer une fois de plus l'écrit en dit. 
La jeune Hanan ne se contente pas du rôle de lectrice, elle devient à son tour narratrice de son propre parcours. La Prière de la peur est plus qu'une œuvre écrite, c'est avant tout une œuvre vocale, une œuvre où les voix féminines se relayent.

Le récit s'achève sur le verbe renaître au futur. Une autre façon de dire qu'il y ara une suite, une relève, et que la fin de La Prière de la peurn'est que le début d'une nouvelle ère. La parole devient délivrante et délivrance pour les protagonistes qui osent la prendre. Elle agit de ce fait sur deux plans, elle sauve et elle est, par la même occasion, sauvée à travers sa continuité assurée par sa transmission. L'élément qui permet cette continuité est le roman qui se voit à son tour renouvelé par le conte.

En mettre en avant trois personnages féminins appartenant à trois générations successives et jouant trois rôles différents et complémentaires, Latifa Ben Mansour reconstitue la chaîne de transmission tout en transformant la transmission orale en transmission écrite par l'introduction d'un personnage conteur, un autre scripteur, et exprime l'altération occasionnée par le passage de l'oral à l'écrit en créant un personnage lecteur.Rassemblés autour du manuscrit, ces trois personnages, qu'on pourrait qualifier de femmes-récits, parviennent à ressouder les maillons de la chaîne de transmission et à pérenniser l'histoire des ancêtres et à la transmettre.

Si l'oralité renvoie au passé, sa fixation par l'écriture l'inscrit dans le présent, et lui assure une continuité dans le futur, assurée dans le texte mansourien par le manuscrit et la nouvelle génération qui assurera la relève.

Ben Mansour puise sa matière littéraire de l'oralité qu'elle revisite et retravaille par et dans l'écriture romanesque à travers les personnages, leurs fonctions, mais aussi par l'éclatement de la construction narrative.

L'introduction de l'oralité dans le texte romanesque ne relèverait pas de la reproduction, mais du réemploi et de la réactualisation littéraire pour de donner un nouveau souffle à ce que l'oubli condamne. En intégrant l'écriture, l'oralité rentre dans un nouveau cycle.

Un rapport d'échange productif se crée entre ces deux pôles : l'oralitéenrichit le texte littéraire par sa diversité, le roman pour sa part épouse les formes orales et devient un support de sauvegarde d'une authenticité vouée à la disparition. L'objectif n'est donc pas de fixer l'oralité par le biais de l'écriture, mais de faire de l'œuvre écrite un espace en mouvement, un espace de mouvement, de communion et de pluralité vocale, lieu de réalisation d'une narration vivante et plurielle à l'image de la viepermettant l'actualisation de l'oralité et sa transmission dans et à travers l'écriture.

L'oralité et l'écriture se disent ou se lisent, elles sont destinées à un public, mais entre les deux se situe l'acte scripturaire, permettant la transformation du dit en écrit, de la voix en graphie, offrant ainsi la possibilité 
de retrouver dans la froideur de l'objet « livre » la chaleur des mots, et tout simplement celle de la vie.

\section{Bibliographie :}

- Mikhail Bakhtine, Esthétique et théorie du roman, Paris, Gallimard, 1978 [1975].

- Mikhaïl Bakhtine, La poétique de Dostoïevski, Paris, Seuil, 1970, [1929, 1963].

- BARTHES Roland, Le Grain de la voix, entretiens, 1962-1980, Paris, Seuil, 1981.

- BEN MANSOUR Latifa, La Prière de la peur, Paris, La Différence, 1997.

- BERTHELOT Francis, Parole et dialogue dans le roman, du discours intérieur au dialogue, Paris, Nathan, 2001.

- BERTRAND Michel, Langue Romanesque et parole scripturale, essai sur Claude Simon, P.U.F, 1987.

- BUTOR Michel, La Balance des fées, in Répertoire I,Paris, Minuit, 1968.

- CALAME-GRIAULE Geneviève, Le renouveau du conte, Paris,CNRS, 1991.

- CARLIER Christophe, La Clef des contes, Ellipses, 1998.

- CHAULET-ACHOUR Christiane et AZZA-BEKKAT Amina, Clefs pour la lecture des récits, Convergences critiques II, Ed. Du Tell, Blida, 2002.

- DALLENBACH Lucien, Le Récit spéculaire, Essai sur la mise en abyme, Paris, Seuil, 1977.

- $\quad$ DE CERTEAU Michel, L'Écriture de l'histoire, Paris, Gallimard, 1975.

- DEJEUX Jean, La littérature féminine de langue française au Maghreb, Ed. Karthala, Paris, 1994.

- GENETTE Gérard, Discours du récit, Seuil, 2007 [1972].

- GOODY Jack, Entre l'oralité et l'écriture, Paris, P.U.F., 1994.

- JEAN Georges, Le pouvoir des contes, Casterman, 1990 [1981].

- KAZI-TAMI Nora Alexandra, Roman africain de langue française au carrefour de l'écrit et de l'oral (Afrique noire et Maghreb), L'Harmattan, 1995.

- KEGLE Christiane, GODIN Richard, Les récits de survivance, modalités génériques et structures d'adaptation au réel, PUF, 2007.

- LALAOUI Fatima Zohra, Écriture de l'oralité et contre discours féminin dans Loin de Médine d'Assia Djebar, Articlein Semen, n 18, De la culture orale à la production écrite: Littérature africaines, 2004.

- $\quad$ TADIE Jean-Yves et Marc, Le sens de la mémoire, Gallimard, 1999. 
- ZUMTHOR Paul, Introduction à la poésie orale, Paris,Seuil, col. Poétique, 1983.

\section{Notes :}

(1)-Latifa Ben Mansour, Entretien in Air France madame, Juin-juillet 1997.

(2)-Latifa Ben Mansour, La Prière de la peur, La Différence, 1997.

(3)-Michel Butor, La Balance des fées, in Répertoire I, éd. de Minuit, 1968, p. 117.

(4)-Georges Jean, Le pouvoir des contes, Casterman, 1990, p.14.

(5)-Idem.

(6)- Christophe Carlier, La Clef des contes, éd. Ellipses, 1998, p. 41.

(7)-Idem.

(8)-Mikhail Bakhtine, Esthétique et théorie du roman, Paris, Gallimard, 1978, p. 181.

(9)-Idem.,p. 102.

(10)- Mikhaïl Bakhtine, La poétique de Dostö̈evski, Paris, Seuil, 1970, p. 188.

(11)-Idem., p. 249.

(12)-Francis Berthelot, Parole et dialogue dans le roman, Paris, Nathan, 2001, p. 122.

(13)- Idem., p. 126. 